\title{
Influence of Autologous and Homologous Blood Transfusion on Interleukins and Tumor Necrosis Factor- $\alpha$ in Peri-operative Patients with Esophageal Cancer
}

\author{
Yue-Li Xing ${ }^{1 *}$, Yan-Chun Wang ${ }^{2}$
}

\begin{abstract}
Objective: To explore the influence of different ways of blood transfusion on the expression levels of interleukins (IL) and tumor necrosis factor- $\alpha$ (TNF- $\alpha$ ) inperi-operative patients with esophageal cancer. Materials and Methods: A total of 80 patients with esophageal cancer who underwent radical operations were selected as study patients and randomly divided into an observation group (treated with autologous blood transfusion) and control group (with homologous blood transfusion). Changes of intra-operative indexes and peri-operative blood indexes, from hemoglobin $(\mathrm{Hb})$ and hematocrit value $(\mathrm{Hct})$, to levels of inflammatory factors like interleukins-6 (IL-6), IL-8, IL-10 and tumor necrosis factor- $\alpha$ (TNF- $\alpha$ ) were compared. Results: Operations for patients in both groups were successfully conducted, and no significant differences in mean surgical duration and intra-operative hemorrhage volume, fluid infusion volume and blood transfusion volume were detected $(p>0.05)$. Compared with values before surgery, $\mathrm{Hb}$ and Het levels decreased significantly while white blood cell count (WBC) increased 1,5 and $7 \mathrm{~d}$ after operation $(p<0.05, p<0.01)$. In addition, $\mathrm{WBC}$ was apparently higher in observation group than in control group 5 and $7 \mathrm{~d}$ after operation $(p<0.01)$. Compared with before surgery, in the observation group, levels of IL-6, IL-8 and IL-10 had no significant differences after operation (P>0.05), but TNF- $\alpha$ level increased y $(p<0.01)$, whereas in control group, IL-6 level had no significant difference ( $p>0.05)$, IL-8 level decreased obviously $(p<0.05)$, IL-10 level increased markedly first and then decreased gradually as time passed but its level remained elevated $(p<0.01)$, and TNF- $\alpha$ level increased first and then decreased, and there was no significant difference $7 \mathrm{~d}$ after operation $(p>\mathbf{0 . 0 5})$. Conclusions: Decreased IL-8 and increased IL-10 levels are two important reasons forimmunosuppression after homologous blood transfusion, whereas autologous blood transfusion can alleviate this while increasing the TNF- $\alpha$ level, which also has potential to improve anti-tumor immunity in the human body.
\end{abstract}

Keywords: Blood transfusion - interleukin - tumor necrosis factor -esophageal cancer

Asian Pac J Cancer Prev, 15 (18), 7831-7834

\section{Introduction}

In 1818, Blundell, an English obstetrician, performed blood transfusion to 11 parturients in order to resolve the massive hemorrhage, in which 5 were successfully treated, which largely improved the development of blood transfusion. In 1901, Landsteiner found the first human blood-group system (ABO blood groups), after which the discovery of the importance of cross-matching of blood between donators and receptors, the application of Sodium Citrate Injection for Anticoagulant and the finding of $\mathrm{RH}$ blood-group system have promoted the fast development of blood transfusion and made it a critical therapeutic method.

At present, blood transfusion had become an important complementary therapy for clinical operations, which can strengthen oxygen-carrying ability of cells and supplement blood volume and the colloid ingredients and coagulation factors of blood. However, there are still many blood transfusion associated complications, like hemolysis, fever, allergy, immunosuppression and infectious diseases induced by virus and bacteria, etc.. As early as 1970s, studies have showed that homologous blood transfusion can lead to blood-transfusion connected immunosuppression (Opelzet al., 1973) and prei-operative blood transfusion can increase the recurrence rate of tumors (Amato et al., 2006). It is estimated that about $0.2 \%$ blood receptors have showed inflammatory responses marked by systematic inflammatory response syndrome due to blood transfusion, which can trigger serious septicemia, lung 
failure and multiple organ failures (Urner et al., 2012). Therefore, blood protection technique receives more and more attentions recently. As one of the blood protection techniques, autologous blood transfusion is gradually applied as a complementary therapy in clinical operation because it can not only avoid various transmission diseases due to blood transfusion, but also prevent homogeneous homologous blood transfusion induced homogeneous immunological responses. However, the influence of autologous blood transfusion on the immunological function of human body is still controversial. In order to explore the influence of different ways of blood transfusion on immunological functions of patients with esophagus carcinoma, this study detected the dynamic changes of inflammatory factors such as interleukins (IL) and tumor necrosis factor- $\alpha$ (TNF- $\alpha$ ), hoping to provide theoretical basis for the rational application of blood transfusion in peri-operative patients with esophagus carcinoma.

\section{Materials and Methods}

\section{General data}

A total of 80 patients who were diagnosed with esophagus carcinoma by gastroendoscope and histopathological detection and received radical operations were selected as study objects, in which there were 44 males and 36 females, aged from 32 years to 68 years, with mean age being $(49.15 \pm 7.23)$ years. Inclusion criteria: Predicative hemorrhage volume $>500 \mathrm{~mL}$; American Society of Anesthesiologists (ASA): phase I II; With well functions of heart and lung; Without hepatorenal function damage and metabolic, respiratory and immunological system diseases; Without drugs that could affect immunological function in peri-operative period, like corticosteroid; Without virus and bacterial infection and without fever before operation. The patients were randomly divided into observation group and control group, 40 cases for each group. There were significant differences in general data such as genders, ages, tumor histological types and patterns $(P>0.05)$, which were comparable.

All therapeutic measures were approved by ethics committees and conducted after Informed Consent Forms were signed by patients or their families.

\section{Methods}

Anesthesia method: General anesthesia was adopted in both groups. Induction routine of intravenous anesthesia: fentanyl $2 \mu \mathrm{g} / \mathrm{kg}$, midazolam $0.04 \mathrm{mg} / \mathrm{kg}$, propofol $1.5 \mathrm{mg} / \mathrm{kg}$ and vecuronium bromide $0.12 \mathrm{mg} / \mathrm{kg}$. Anesthesiamaintenance: continuous intravenous injection of fentanyl $0.1 \sim 0.2 \mathrm{mg} /(\mathrm{kg} \cdot \mathrm{min})$ and remifentanil $0.05 \sim 0.2 \mathrm{mg} /(\mathrm{kg} \cdot \mathrm{min})$. Patient-controlled analgesia: intravenous injection of fentanyl $6 \sim 8 \mu \mathrm{g} / \mathrm{mL}$.

Blood transfusions: Observation group was treated with autologous blood transfusion, for which blood tests were given immediately after patients were hospitalized, and $400 \mathrm{~mL}$ autologous blood was collected in advance from patients with hemoglobin $(\mathrm{Hb})>110 \mathrm{~g} / \mathrm{L}$ one week before operation and then stored at $4{ }^{\circ} \mathrm{C}$ for utilization. After operations were started, full automatic blood salvage machine (Type: Cell Saver-5, produced by American Haemonetics company) was used to absorb the lost blood in operative field during operation. Meanwhile, $30000 \mu / 1$ $000 \mathrm{~mL}$ normal saline (NS) containing heparin was added at $60 \mathrm{drops} / \mathrm{min}$ for anti-coagulation. Then the blood was filtered, washed, centrifuged and stored in blood bag for use. The blood was used when $\mathrm{HB}<100 \mathrm{~g} / \mathrm{L}$ and hematocrit value $(\mathrm{Hct})<0.3$. Control group was given appropriate homologous blood according to the hemorrhage volume and vital sign of patients, in which autologous blood was not collected in advance before operation and no lost blood in operative field was recovered during operation.

\section{Sample collection and detection}

Sample collection: $5 \mathrm{~mL}$ fasting venous blood $1 \mathrm{~d}$ before operation and 1, 5 and $7 \mathrm{~d}$ after operation was collected and centrifuged at $3500 \mathrm{r} / \mathrm{min}$ for $15 \mathrm{~min}$ at $4^{\circ} \mathrm{C}$. Supernatant was collected and stored at $-80^{\circ} \mathrm{C}$ for utilization.

Sample detection: Double-antibody enzyme-linked immunosorbent assasy (ELISA) was adopted to detect inflammatory factors, such as interleukin-6 (IL-6), IL-8, IL-10 and tumor necrosis factor- $\alpha$ (TNF- $\alpha)$. ELISA imark (American Bio-Rad Company) was applied with all kits being purchased from Cell-Bio Biotechnology Co., Ltd. All procedures were conducted strictly according to the instructions on kits.

\section{Observational indexes}

Intra-operative indexes (hemorrhage volume, fluid infusion volume and blood transfusion volume), peripheral blood indexes ( $\mathrm{Hb}$, Hct and $\mathrm{WBC}$ ) and peri-operative inflammatory factors (IL-6, IL-8, IL-10 and TNF- $\alpha$ ) in both groups were observed.

\section{Statistical data analysis}

SAS 9.3 software package was applied for all data analysis. Measurement data was expressed by $(\mathrm{x} \pm \mathrm{s})$, in which measurement data with normal distribution between two groups was compared by t-test while those with nonnormal distribution between two groups by rank-sum test, and comparisons among multiple groups by Chi-squared test. $P<0.05$ was considered to be statistically significant.

\section{Results}

Comparisons of intra-operative indexes between two groups

Operations for patients of both groups were successfully completed, and there was no significant difference in mean surgical duration and intra-operative hemorrhage volume, fluid infusion volume and blood transfusion volume $(P>0.05)$ (Table 1).

Changes of peripheral blood before and after operations in both groups

Compared with operation before, $\mathrm{Hb}$ and Hct levels decreased evidently while WBC increased markedly 1 , 5 and $7 \mathrm{~d}$ after operation $(P<0.05, P<0.01)$. In addition, WBC was apparently higher in observation group than in control group 5 and $7 \mathrm{~d}$ after operation $(P<0.01)$. However, 
Table 1. Comparisons of Intra-operative Indexes Between Two Groups $(\mathrm{x} \pm \mathrm{s})$

\begin{tabular}{lcccc}
\hline & Surgical duration/min & Hemorrhage volume $/ \mathrm{mL}$ & Fluid infusion volume $/ \mathrm{mL}$ & Blood transfusion volume $/ \mathrm{mL}$ \\
\hline Observation group & $327.19 \pm 42.35$ & $1601.25 \pm 261.45$ & $2180.00 \pm 274.37$ & $1190.00 \pm 194.39$ \\
Control group & $324.86 \pm 39.67$ & $1673.75 \pm 271.61$ & $2230.00 \pm 292.58$ & $1210.00 \pm 221.48$ \\
t value & 0.25 & -1.22 & -0.79 & -0.43 \\
$P$ vaule & 0.8002 & 0.2276 & 0.4329 & 0.6689 \\
\hline
\end{tabular}

Table 2. Changes of Peripheral Blood before and after Operations in Both Groups $(\mathbf{x} \pm \mathbf{s})$

\begin{tabular}{lcccc}
\hline & $\begin{array}{c}\text { Operation } \\
\text { before }\end{array}$ & $\begin{array}{l}1 \mathrm{~d} \text { after } \\
\text { operation }\end{array}$ & $\begin{array}{l}5 \mathrm{~d} \text { after } \\
\text { operation }\end{array}$ & $\begin{array}{l}7 \mathrm{~d} \text { after } \\
\text { operation }\end{array}$ \\
\hline $\mathrm{Hb}(\mathrm{g} / \mathrm{L})$ & & & & \\
Observation group & $126.4 \pm 6.9$ & $117.1 \pm 5.2^{* *}$ & $110.2 \pm 4.9^{* *}$ & $115.4 \pm 5.0^{* *}$ \\
Control group & $129.3 \pm 7.1$ & $116.3 \pm 5.7^{* *}$ & $109.8 \pm 4.6^{* *}$ & $113.9 \pm 4.9^{* *}$ \\
$\mathrm{Hct}(\%)$ & & & & \\
Observation group & $40.2 \pm 3.5$ & $35.4 \pm 3.9^{* *}$ & $30.2 \pm 3.4^{* *}$ & $31.3 \pm 4.1^{* *}$ \\
Control group & $40.7 \pm 3.7$ & $33.7 \pm 4.0^{* *}$ & $29.7 \pm 3.3^{* *}$ & $30.5 \pm 3.4^{* *}$ \\
WBC (109/L) & & & & \\
Observation group & $5.4 \pm 0.6$ & $7.6 \pm 0.6^{* *}$ & $6.9 \pm 0.5^{* * \# *}$ & $7.0 \pm 0.7^{* * \# \#}$ \\
Control group & $5.7 \pm 0.4$ & $7.9 \pm 0.8^{* *}$ & $5.9 \pm 0.4^{*}$ & $6.1 \pm 0.4^{* *}$ \\
\hline
\end{tabular}

Compared with operation before, $* P<0.05, * * P<0.01$; Compared with synchronous control group, ${ }^{\# \#} P<0.01$

the indexes at other time points had no significant differences between two groups $(P>0.05)$ (Table 2).

Changes of inflammatory factors before and after operations in both groups

Compared with operation before, in observation group, IL-6, IL-8 and IL-10 levels had no significant differences after operation $(P>0.05)$ whereas TNF- $\alpha$ level increased gradually after operation and reached its peak $5 \mathrm{~d}$ after operation and then the level began to decrease, but was still evidently higher than operation before $(P<0.01)$. Compared with operation before, in control group, IL-6 level had no significant difference $(P>0.05)$, IL-8 level decreased obviously $(P<0.05, P<0.01)$, IL-10 level increased markedly and then decreased gradually, but was still higher than the level before operation $(P<0.01)$, and TNF- $\alpha$ level increased gradually after operation and reached its peak $1 \mathrm{~d}$ after operation, which was still evidently higher than the level before operation $(P<0.01)$, then the level decreased gradually and there was no significant difference between the level $7 \mathrm{~d}$ after operation and that before operation $(P>0.05)$. Comparison between two groups showed that there was no significant difference in IL-6 level at each time points $(P>0.05)$, whereas the differences were significant in the rest indexes between at each synchronous time points $(P<0.01)$ (Table 3$)$.

\section{Discussion}

At present, treatments for multiple cancers (including esophagus carcinoma) mainly focus on surgical excision (Chen et al 2013; Zhang et al 2013; Tatematsu et al 2013), but the general anesthesia, surgical therapies and traumas induced postoperative stress responses can trigger postoperative immunological dysfunction to cancer patients, which may aggravate the existed immunosuppression (Guo et al., 2014). In addition, intra-operative blood transfusion is an indispensible complementary therapy due to the large traumas, longer duration and more hemorrhage volume in the operations for cancer patients, which can immediately remedy the intraoperative anemia and insufficient blood volume. However, blood transfusion-associated immunosuppression may further aggravate the postoperative immunosuppression of cancer patients, which will enable residual and exfoliative tumor cells escape from immunological surveillance, leading to recurrence and distant metastasis of tumors (De Oliveira et al., 2012; Meng et al., 2013). A study found that autologous blood transfusion could improve the postoperative immunological function and protect organs of cancer patients (Han et al., 2011; Stachura et al., 2011). This study mainly detect the dynamic changes of IL-6, IL-8, IL-10 and TNF- $\alpha$ levels before and after operations so as to explore the influence of autologous and homologous blood transfusions on the immunological function of patients with esophagus carcinoma.

TNF- $\alpha$ and interleukins are important cellular factors in human body. TNF- $\alpha$ has multiple biological activities, which involves in inflammatory and immunological responses and has anti-tumor immunological functions. Meanwhile, it also participates in pathological processes such as endotoxin shock. Additionally, TNF- $\alpha$ also has multiple functions of anti-inflammation and killing tumor cells due to its synergistic action with other bioactive factors (Chen et al., 2010; Fujiwara et al., 2013; Su et al., 2013). Previous research discovered that TNF- $\alpha$ could kill the residual tumors through inducing cell

Table 3. Changes of Inflammatory Factors before and after Operations in Both Groups $(\mathrm{x} \pm \mathrm{s}, \mathrm{pg} / \mathrm{mL})$

\begin{tabular}{llcccc}
\hline Indexes & Groups & \multicolumn{3}{c}{ Peri-operative period } \\
\cline { 3 - 6 } & & Operation before & $1 \mathrm{~d}$ after operation & $5 \mathrm{~d}$ after operation & $7 \mathrm{~d}$ after operation \\
\hline IL-6 & Observation group & $172.32 \pm 69.98$ & $166.53 \pm 70.28$ & $169.85 \pm 67.22$ & $170.01 \pm 65.97$ \\
& Control group & $176.41 \pm 70.36$ & $159.38 \pm 67.25$ & $162.76 \pm 65.27$ & $171.03 \pm 71.14$ \\
IL-8 & Observation group & $16.31 \pm 3.57$ & $17.74 \pm 4.75^{\# \#}$ & $16.95 \pm 2.01^{\# \#}$ & $16.49 \pm 4.21^{\# \#}$ \\
& Control group & $15.92 \pm 3.46$ & $14.43 \pm 3.16^{*}$ & $14.28 \pm 3.08^{*}$ & $13.87 \pm 2.46^{* *}$ \\
IL-10 & Observation group & $7.38 \pm 2.95$ & $8.49 \pm 2.14^{\# \#}$ & $8.07 \pm 1.76^{\# \#}$ & $7.34 \pm 2.51^{\# \#}$ \\
& Control group & $7.59 \pm 1.07$ & $17.16 \pm 3.04^{* *}$ & $14.67 \pm 3.85^{* *}$ & $9.51 \pm 2.97 * *$ \\
TNF- $\alpha$ & Observation group & $1.26 \pm 0.54$ & $1.97 \pm 0.66^{* * \# \#}$ & $5.85 \pm 1.03^{* * \# \#}$ & $3.77 \pm 1.10^{* * * \# \#}$ \\
& Control group & $1.23 \pm 0.69$ & $3.19 \pm 0.57^{* *}$ & $2.01 \pm 0.48^{* *}$ & $1.28 \pm 0.71$ \\
\hline
\end{tabular}

Compared with operation before, ${ }^{*} P<0.05,{ }^{*} * P<0.01$; Compared with synchronous control group, ${ }^{\# \#} P<0.01$ 
apoptosis, and many anti-tumor agents (cisplatin, etc.) could achieve their anti-tumor action by increasing TNF- $\alpha$ level (Kondo et al., 2006). IL-10 can inhibit the proliferation transplantation and immune damage effect of human body to tumors via suppressing multiple cytokines that could kill tumor cells. In addition, IL-10 could also improve the metastasis and proliferation of tumors by strengthening the immuniological escape function of tumor cells (O'Garra et al., 2008). IL-6 and IL-8, as two critical cytokines in activating anti-inflammatory responses, can promote the exudation and chemotixas of inflammatory cells and activate inflammatory cells, the increase of whose activities could inhibit and remove bacteria and increase the patients' anti-inflammatory and wound-healing abilities.

In this study, two blood transfusion methods were applied to reduce the rate of homologous blood transfusion in patients with atuologous blood transfusion, which meant the autologous blood salvage technique in predeposited autotransfusion. The results demonstrated that TNF- $\alpha$ level increased evidently after operation in observation group, which might be of great significance for the anti-tumor effect of human body. However, IL-10 level increased markedly in control group, especially 1 $\mathrm{d}$ after operation, and the level at each time points was apparently higher than that in observation group at the same time point, which might inhibit the activation of $\mathrm{T}$ lymphocytes, and even suppress the release of some cytokines, bringing about decreased immunological surveillance function of human body. Simultaneously, IL-8 level decreased obviously in control group, suggesting that the immunological function was inhibited to some extent in patients with esophagus carcinoma. Previous study indicated that autologous blood transfusion could increase IL-6 level (Schroeder et al., 2007). But in this study, IL-6 level had no significant difference before and after operations in both groups, which was predicated to be associated with the study objects because the cancer patients themselves had certain degree of immunological dysfunction, but the influence of autologous blood transfusion on IL-6 level in patients with esophagus carcinoma needs to be further studied.

To sum up, the decreased IL-8 level and increased IL-10 level are critical potential factors inducing immunosuppression after homologous blood transfusion, and autologous blood transfusion can alleviate immunosuppression while increase TNF- $\alpha$ level, which may improve the anti-tumor effect of patients with esophagus carcinoma.

\section{References}

Amato A, Pescatori M (2006). Perioperative blood transfusions for the recurrence of colorectal cancer. Cochrane Database Syst Rev, 25, CD005033.

Chen SB, Weng HR, Wang G, et al (2013). Surgical treatment for early esophageal squamous cell carcinoma. Asian Pac J Cancer Prev, 14, 3825-30.

Chen X, Oppenheim JJ (2010). TNF- $\alpha$ : an activator of CD4+ FoxP3+ TNFR2+ regulatory T cells. Curr Dir Autoimmun, 11, 119-34.
De Oliveira GS, Schink JC, Buoy C, et al (2012). The association between allogeneic perioperative blood transfusion on tumour recurrence and survival in patients with advanced ovarian cancer. Transfus Med, 22, 97-103.

Fujiwara Y, Shiba H, Iwase R, et al (2013). Inhibition of nuclear factor kappa-B enhances the antitumor effect of combination treatment with tumor necrosis factor-alpha gene therapy and gemcitabine for pancreatic cancer in mice. $\mathrm{J} \mathrm{Am} \mathrm{Coll}$ Surg, 216, 320-32.

Guo JR, Feng X, Jin XJ, et al (2014). Impact of allogenic and autologous transfusion on immune function in patients with tumors. Asian Pac J Cancer Prev, 15, 467-74.

Han CB (2011). Effect of immunoglobulin on autologous blood transfusion in patients with colorectal cancer. J Clin Hematol, 24, 597-8.

Kondo K, Yamasaki S, Sugie T, et al (2006). Cisplatin-dependent upregulation of death receptors 4 and 5 augments induction of apoptosis by TNF-related apoptosis-inducing ligand against esophageal squamous cell carcinoma. Int J Cancer, 118, 230-42.

Meng J, Lu XB, Tang YX, et al (2013). Effects of allogeneic blood transfusion in patients with stage II colon cancer. Asian Pac J Cancer Prev, 14, 347-50.

O'Garra A, Barrat FJ, Castro AG, et al (2008). Strategies for use of IL-10 or its antagonists in human disease. Immunol Rev, 223, 114-31.

Opelz G, Sengar DP, Mickey MR, et al (1973). Effect of blood transfusions on subsequent kidney transplants. Transplant Proc, 5, 253-9.

Schroeder S, von Spiegel T, Stuber F, et al (2007). Interleukin-6 enhancement after direct autologous retransfusion of shed thoracic blood does not influence haemodynamic stability following coronary artery bypass grafting. Thorac Cardiovasc Surg, 55, 68-72.

Stachura A, Król R, Poplawski T, et al (2011). Transfusion of intra-operative autologous whole blood: Influence on complement activation and interleukin formation. Vox Sang, 100, 239-46.

Su B, Cengizeroglu A, Farkasova K, et al (2013). Systemic $\mathrm{TNF} \alpha$ gene therapy synergizes with liposomal doxorubicine in the treatment of metastatic cancer. Mol Ther, 21, 300-8.

Tatematsu N, Park M, Tanaka E, et al (2013). Association between physical activity and postoperative complications after esophagectomy for cancer: a prospective observational study. Asian Pac J Cancer Prev, 14, 47-51.

Urner M, Herrmann IK, Buddeberg F, et al (2012). Effects of blood products on inflammatory response in endothelial cells in vitro. PloS One, 7, e33403.

Zhang BH, Cheng GY, Xue Q, et al (2013). Clinical outcomes of basaloid squamous cell carcinoma of the esophagus: a retrospective analysis of 142 cases. Asian Pac J Cancer Prev, 14, 1889-94. 